\title{
Enzymatic production of dietary nucleotides from low-soluble purine bases by an efficient, thermostable and alkali-tolerant biocatalyst
}

\author{
J.Del Arco, J.Cejudo-Sanches, I.Esteban, V.J.Clemente-SuárezaD.Hormigo, \\ A.Perona, Jesus Fernandez Lucas
}

\begin{abstract}
Traditionally, enzymatic synthesis of nucleoside-5'-monophosphates (5'-NMPs) using low water-soluble purine bases has been described as less efficient due to their low solubility in aqueous media. The use of enzymes from extremophiles, such as thermophiles or alkaliphiles, offers the potential to increase solubilisation of these bases by employing high temperatures or alkaline $\mathrm{pH}$. This study describes the cloning, expression and purification of hypoxanthine-guanine-xanthine phosphoribosyltransferase from Thermus thermophilus (TtHGXPRT). Biochemical characterization indicates TtHGXPRT as a homotetramer with excellent activity and stability across a broad range of temperatures $\left(50-90^{\circ} \mathrm{C}\right)$ and ionic strengths $(0-$ $500 \mathrm{mM} \mathrm{NaCl}$ ), but it also reveals an unusually high activity and stability under alkaline conditions ( $\mathrm{pH}$ range 8-11). In order to explore the potential of TtHGXPRT as an industrial biocatalyst, enzymatic production of several dietary $5^{\prime}-\mathrm{NMPs}$, such as $5^{\prime}-\mathrm{GMP}$ and $5^{\prime}-\mathrm{IMP}$, was carried out at high concentrations of guanine and hypoxanthine.
\end{abstract}

\section{Keywords}

Enzymatic Synthesis, Food Industry, 6-Oxopurine Phosphoribosyltransferases, Thermophiles, Alkaliphiles 